\title{
The Role of Vague Categories in Semantic and Adaptive Web Interfaces
}

\author{
Miguel-Ángel Sicilia \\ Computer Science Department, Carlos III University, \\ Av. Universidad, 30 - 28911 Leganés (Madrid), Spain \\ msicilia@inf.uc3m.es
}

\begin{abstract}
Current Semantic Web technologies provide a logic-based framework for the development of advanced, adaptive applications based on ontologies. But the experience in using them has shown that, in some cases, it would be convenient to extend its logic support to handle vagueness and imprecision in some way. In this paper, the role of vagueness in the description of Web user interface characteristics is addressed, from the viewpoint of the design of adaptive behaviors that are connected to such descriptions. Concretely, vague descriptions combined with quantified fuzzy rules and flexible connectors are described, and their usefulness is illustrated through preference modeling, filtering and adaptive linking scenarios.
\end{abstract}

\section{Introduction}

The vision of a Semantic Web [4] has recently attracted the attention of researchers coming from various disciplines. The essential element of that vision is the provision of meta-information for Web resources, in a machineunderstandable form, so that it can be shared and processed by automated tools, thus enabling a new generation of 'intelligent' applications. Consequently, both (a) a lingua franca for annotation, and also (b) a number of consensual terminologies are required to guarantee a common understanding of such metainformation. Description Logics (DL) [2] and formal ontologies expressed using them [11] have been proposed as the response for requirements (a) and (b) respectively [3]. In the last decade, a substantial amount of theoretical and applied research regarding DL has been carried out [2], resulting in logics bearing a good compromise between expressive power and computational complexity. Nonetheless, since the publication of McCarthy's seminal papers on epistemological problems - see, for example, [17] -, any problem of artificial intelligence (AI) is considered to comprise an epistemological part, and a heuristic part. The former is concerned with the representation of facts about the world as are available to us, while the latter is concerned with the algorithms required to make AI computations feasible. Therefore, despite the heuristic appropriateness of current DLs to many common problems, it still remains necessary an inquiry about its epistemological adequacy for the kinds of knowledge that should be encoded in Semantic Web applications. 
Here we are mainly concerned with the epistemological adequacy of Semantic Web applications, with regards to vagueness as a human categorization characteristic [18]. More concretely, previous experiences [8,16,19,9] have lead us to consider vague categories as an essential problem component in user modeling and adaptation regarding characteristics of human's perceptions of Web user interfaces. These aspects include descriptions of Web user interface (UI) elements - like for example, sizes, density, granularity and link structure - , not directly related to the content (i.e. the meaning) of the page. This characteristic makes UI descriptions domain-independent, and in consequence, of a wide applicability, orthogonal and complementary to annotations regarding content description or authorship. In addition, these UI descriptions are important both for the representation of user models and to express adaptation rules. For example, user preferences regarding granularity may be expressed as "user U prefers long descriptions", where long represents a fuzzy subset defined on the length (measured in words, for example) of content items, and a rule like "if student is novice then provide him with simple explanations" enables adapting the density of explanation items using the imprecise simple category. It may be argued that collapsing these kind of vague concepts into discrete enumerations (e.g. \{very long, long, medium, short, very short \} for Web pages) could solve the problem from a practical perspective. But previous research on membership elicitation of simple Web characteristics [9] provides evidence against such claim, since elicited fuzzy subsets for a given category are neither regular in function shape, nor equally spaced in the domain of definition. In addition, some adaptive hypermedia technologies [6] are adequate for partial truth-transfer inferencing. For example, one of the most typical link navigation adaptive technologies is link annotation. If the attribute of the link to be adapted is of a variable nature like font size (or font color), a rule with a consequent part like "change font-size of link L to large" may produce different "large" fonts according to the degree of activation of some fuzzy antecedent. The concept of personalized fuzzy links [21] are a different formulation for that concept of imprecise adaptiveness.

Previous research have addressed the integration of vague concepts into crisp description logics $[25,26,12]$, resulting in what can be called fuzzy description logics (fDL). Retrieval of multimedia objects [24] and electronic commerce [12] have been mentioned as application areas for fDLs. In this paper, we describe vagueness in UI descriptions as a component of a Semantic Web application, with the objective of providing a framework for adaptation. A number of useful imprecision handling mechanisms that can be integrated with DL are described, without the aim of studying their formal logical properties, that are left to future work. The motivation for those mechanisms is of a practical nature, and consequently, concrete examples we have faced with are provided as well.

The rest of this paper is structured as follows. In Section 2, a general framework for the vague semantic description of adaptive Web user interfaces is sketched. Section 3 illustrates the applicability of such models for concrete adaptive techniques. Finally, conclusions and future research directions are provided in Section 4. 


\section{Vagueness in Adaptive, Semantic Web Interface Models}

The concepts of Adaptive Web [7] and Semantic Web can be considered as two perspectives of the same vision of a prospective Web. While the former one focus on tailoring a hypermedia space to the heterogeneity of user goals, knowledge and interests, the latter one takes a broader view, concentrating on the availability of shared knowledge representations and a common technological support for the development of advanced applications — including adaptive ones - . From here on, we'll follow the assumption that both research areas will converge in the future, and therefore, DLs, perhaps extended or combined with other paradigms, will form the basis of Adaptive-Semantic Web sites. In addition, we take the $\mathcal{A} \mathcal{L C}_{F H}$ fuzzy description logic described in [12] (which in turn is an extension of the Fuzzy- $\mathcal{A L C}$ defined in [25]) as a point of departure for the discussion of practical aspects of vague semantics in user interface descriptions.

In $\mathcal{A} \mathcal{L C}_{F H}$, concepts are expressions that describe, by means of roles, the properties of a set of individuals. Given that $C$ and $D$ are concepts, $M$ is a concept modifier, and $R$ is a role, we have the following syntax rules:

$C, D \longrightarrow$

$$
\begin{aligned}
& \top \text { (top concept) } \\
& \perp \mid \text { (bottom concept) } \\
& A \mid \text { (primitive concept) } \\
& R \mid \text { (primitive role) } \\
& C \sqcap D \mid \text { (concept conjunction) } \\
& C \sqcup D \mid \text { (concept disjunction) } \\
& \neg C \mid \text { (concept negation) } \\
& M A \mid \text { (concept modification) } \\
& \forall R . C \mid \text { (universal quantification) } \\
& \exists R . C \mid \text { (existential quantification) }
\end{aligned}
$$

The semantics of $\mathcal{A L C}_{F H}$ are based on the following interpretations, where $\eta_{M}$ is a membership modifier (this category is intended to model fuzzy hedges like $V E R Y$ or $F A I R L Y)$ :

$$
\begin{gathered}
\top^{\mathcal{I}}(d)=1 \quad \forall d \in \Delta^{\mathcal{I}} \\
\perp^{\mathcal{I}}(d)=0 \quad \forall d \in \Delta^{\mathcal{I}} \\
A^{\mathcal{I}}: \Delta^{\mathcal{I}} \rightarrow[0,1] \\
R^{\mathcal{I}}: \Delta^{\mathcal{I}} \times \Delta^{\mathcal{I}} \rightarrow[0,1] \\
(C \sqcap D)^{\mathcal{I}}(d)=\min \left\{C^{\mathcal{I}}(d), D^{\mathcal{I}}(d)\right\} \\
(C \sqcup D)^{\mathcal{I}}(d)=\max \left\{C^{\mathcal{I}}(d), D^{\mathcal{I}}(d)\right\} \\
(\neg C)^{\mathcal{I}}(d)=1-C^{\mathcal{I}}(d) \\
(M A)^{\mathcal{I}}(d)=\eta_{M}\left(A^{\mathcal{I}}(d)\right) \\
(\forall R . C)^{\mathcal{I}}(d)=i n f_{d^{\prime} \in \Delta^{\mathcal{I}}}\left\{\max \left\{1-R^{\mathcal{I}}\left(d, d^{\prime}\right), C^{\mathcal{I}}\left(d^{\prime}\right)\right\}\right\} \\
(\exists R . C)^{\mathcal{I}}(d)=\sup _{d^{\prime} \in \Delta^{\mathcal{I}}}\left\{\min \left\{R^{\mathcal{I}}\left(d, d^{\prime}\right), C^{\mathcal{I}}\left(d^{\prime}\right)\right\}\right\}
\end{gathered}
$$

A (fuzzy) knowledge base $\Sigma$ is the union of a finite set of fuzzy assertions $\Sigma_{A}$ (often called "A-box") and a finite set of fuzzy terminological axioms $\Sigma_{T}$ (often called "T-box"). Terminological axioms are either fuzzy specializations $A \tilde{\leq} B$ or fuzzy concept definitions $A \cong C$. Fuzzy assertions take the forms sum- 
marized in expression (1), where $n \in[0,1)$. For example, $\langle u: V I P \geq 0.8\rangle$ may be interpreted as "user u belongs to the VIP user group to an extent greater or equal than $80 \%$ ", and $\langle(u, p)$ : interestedIn $\leq 0.3\rangle$ may be interpreted as "the interest of user $\mathrm{u}$ in product $\mathrm{p}$ is lower or equal than $30 \%$ ".

$$
\left\langle\left\{\begin{array}{c}
a: C \\
(a, b): R
\end{array}\right\}\left\{\begin{array}{l}
> \\
< \\
\leq \\
\geq \\
=
\end{array}\right\} n\right\rangle
$$

Given the above definitions, the model of an adaptive hypermedia application can be logically described by means of $\mathcal{A L C}_{F H}$ as a knowledge base including the main elements that comprise the common architecture of such systems [27], extended to cope with imprecision in its principal constructs [22]. The main components of such architecture for a given system $\mathcal{A H}$ can be summarized in the four elements denoted in (2).

$$
\mathcal{A H}=\prec H M, D M, U M, A M \succ
$$

The hypermedia model (HM) describes the structure of the hypermedia application (Web applications are a concrete form of them), including contents, nodes and links. The domain model (DM) describes the vocabulary that is used for each concrete application, for example, an online bookstore will provide descriptions for books or CDs, while a educational Web application will have a DM including the concepts that are intended to be taught. The user model contains the description of the users and user categories of the application. Finally, the adaptation model - which is of a different nature to previous one - contains the description of the adaptive behaviors that must be carried out, in terms of the other models. It should be noted that expression (2) is only significant for ease of understanding, since the four models are actually tightly integrated. For example, preferences about books are a relationship traversing DM and UM, and adaptive behaviors in most cases tailor elements in the HM to information in UM and possibly also in DM. All the (sub-)models in (2) — with the possible exception of $\mathrm{AH}$, that usually includes also rules or procedural formalism - can be described by a $\mathcal{A} \mathcal{L C}_{F H}$ knowledge base $\Sigma_{\mathcal{A H}}$.

In the rest of this section, three concrete manifestations of vagueness that arise in the just described Adaptive-Semantic Web Model are described. For brevity's sake, we'll describe the concepts or relationships pertaining to the above models as they appear in the examples provided below. It should be noted also that, although the focus of this paper is on imprecise descriptions of user interfaces, these manifestations also apply to many cases of content description.

\subsection{Vague Facts as Automated Assertions}

Asserting facts according to the syntax in expression (1) may be unpractical in many situations. Membership functions of vague categories are a way to express 
fuzzy degrees of belonging that can be used instead. These membership functions can be obtained from experts as approximations, or they can be obtained directly from users, by mean of existing membership elicitation techniques [5]. In the context of Web applications, a number of factors influencing the overall usability of the page have been studied [14], including amount of text, number and type of links and images, color use and page length. These characteristics can be modeled from user studies as collections of linguistic labels, as described in [9].

Given that membership functions are defined on a concrete (crisp) type of elements $^{1}$, we can state that the assertion of an instance may entail the immediate generation ${ }^{2}$ of $k$ other assertions belonging to predefined vague categories $c_{1}, c_{2}, \ldots c_{k}$ defined by their respective membership functions $\mu_{c_{1}}, \mu_{c_{2}}, \ldots \mu_{c_{k}}$. Each $\mu_{j}$ will have as domain of definition one or several discrete or continuous characteristics of the given type of instance $B$, that is, they will take the form $\mu_{c_{j}}: f^{1}(x) \times f^{2}(x) \cdots \times f^{z}(x) \rightarrow[0,1]$ with $x: C$ and being each $f^{j}(x)$ a function transforming one or several primitive-type characteristics (e.g. relations from $C$ to Integer) of a element belonging to $C$. For example, Figure 1 shows an approximation (using gaussian functions $g(x)=e^{-\left(\frac{x-c}{d}\right)^{2}}$ ) for the resulting membership functions short, medium, long and very-long (from left to right) described in [9]. These functions express the human understanding of the linguistic characterization of Web page length categories, for example, $\mu_{\text {LongPage }}: f^{l}(x) \rightarrow[0,1]$ given that $x: W e b P a g e$ and $f^{l}: C \rightarrow \mathbb{N}$ (defined on the domain of lengths in pixels).

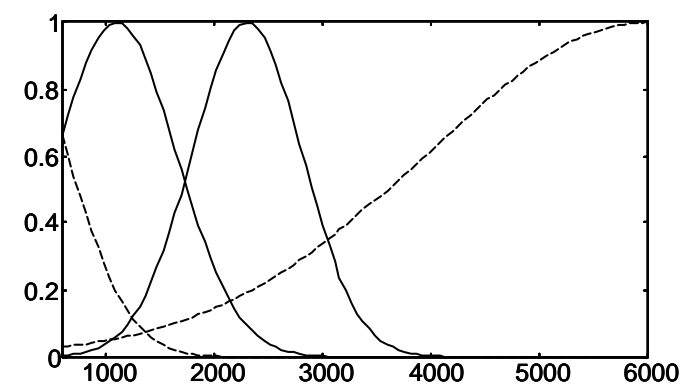

Fig. 1. Membership functions related to Web page length

This way, the assertion of an instance $\langle u: W e b P a g e=1\rangle$ will produce four automatic assertions corresponding to each of the functions in Figure 1 (like, for example, $\langle u: L o n g=0.6\rangle)$. Many user interface related descriptions — including granularity, sizes, semantic density and number of links or images - should be describe this way, since they qualify as imprecise human cognitive categories

\footnotetext{
${ }^{1}$ This may not be the case if we consider higher-order fuzzy sets, but we'll not deal with them here

${ }^{2}$ Of course, heuristic adequacy may preclude this eager approach, resulting in lazy asserting behaviors.
} 
according to [18]. These functions may also be $n$-dimensional, for example, a category $\mu_{G T F}: f^{\# w}(x) \times f^{\# c w c}(x): \rightarrow[0,1]$ may map word counts and column word counts to a degree of belonging to category of "well text-formatted" Web pages.

From the viewpoint of $\mathcal{A} \mathcal{L C}_{F H}$, these automatic assertions do not entail semantic changes, but enable a nice alternative to the built-in hedges $\eta_{M}$ described above. This alternative is that of introducing a explicit term $M_{C}$ instead of introducing $C$, and relying on the logic-based interpretation of modifier $M$. In some cases, this enables being consistent with existing elicitation studies that question the validity of some "classical" hedges. For example, the $V E R Y$ hedge is usually represented simply as $\mu_{V E R Y-A}(x)=\mu_{A}(x)^{2}$, while several experimental studies (summarized in [5]) report a shift in the membership function.

\subsection{Integration of Fuzzy Rules with Quantifiers}

Rules can be combined with description logics enabling forms of hybrid reasoning [1]. Conventional fuzzy rules and well-known fuzzy inferencing mechanisms can be integrated with $\mathcal{A L C}_{F H}$ in a straightforward manner. But fuzzy quantified rules entail a consideration for the cardinality of concepts that is not realized in interpretations of DLs as the above described $\mathcal{I}$. A fuzzy quantified rule can be denoted syntactically in two basic forms, absolute and relative, expressed respectively in (3) and (4). Absolute quantification refers to a single concept like in "many long pages", while relative quantification represent a proportion like in "most long pages are dense". Zadeh' $\sigma$-count operator [29] is one of the possible operationalizations [10] of fuzzy quantification, that we'll use here for simplicity, such that $\sigma-\operatorname{count}(C)=\sum_{x \in C} \mu_{c}(x)$ and $\sigma-\operatorname{count}(C / D)=\frac{\sigma-\operatorname{count}(C \cap D)}{\sigma-\operatorname{count}(D)}$. Details of the application of quantifiers to adaptive hypermedia can be found in [19].

$$
\begin{gathered}
Q c \approx \mu_{Q}(\sigma-\operatorname{count}(c)) \\
Q b \text { are } c \approx \mu_{Q}(\sigma-\operatorname{count}(c / b))
\end{gathered}
$$

One possible way of integrating quantified expressions in languages like $\mathcal{A} \mathcal{L} \mathcal{C}_{F H}$ is treating the result of the quantification as an special assertion. In the absolute case, that assertion has the general form $\langle Q: D=k\rangle$, where $D$ is the concept expression being quantified and $k$ the (current) compatibility grade with quantifier $Q$ similar to an instance assertion. In the relative case, two similar assertions, $\left\langle Q^{\prime}: C \sqcap D=k\right\rangle$ and $\left\langle Q^{\prime \prime}: D=k\right\rangle$, where $C$ is the base concept expression, so that rules can compute the relative count from them.

For example, we can formalize the concept involved in quantified rule 1 as $\exists$ belongs. $\{x\}^{3}$, given that $\langle x:$ WebSite $=1\rangle$ and belong is used to determine the pages in a site. Then, for a given definition of quantifier $M A N Y$ - usually a concrete S-shaped function — an "special" assertion like:

\footnotetext{
${ }^{3}$ The syntax $\{x\}$ to denote a concept is an extension to $\mathcal{A L C}$.
} 


$$
\langle M A N Y: \exists \text { belongs. }\{x\}=0.6\rangle
$$

may be produced (automatically or on demand). This case operates on a crisp set, but vague set expressions of arbitrary complexity can be used instead, for example, the assertion matching the antecedent of rule 2 can be represented as $\langle M A N Y$ : Long $\sqcap$ LOvercrowded $\sqcap \exists$ belongs. $\{x\}=0.4\rangle$.

Example Rule 1 if Web Site X has many pages then "some corrective action"

Example Rule 2 if many of the Web pages of Web Site $X$ are long and link overcrowded then "some corrective action"

A relative quantified expression like "most short pages are dense" may be expressed as the pair of assertions $\left\langle M O S T^{\prime}:\right.$ WebPage $\sqcap$ Long $\sqcap$ Dense $\left.=0.4\right\rangle$ and $\left\langle M O S T^{\prime \prime}:\right.$ WebPage $\sqcap$ Long $\left.=0.6\right\rangle$. Since WebPage is a crisp category, it's used as a filter for both expressions.

\subsection{Flexible Modes of Conjunction}

The conjunction of two concepts in $\mathcal{A L C}_{F H}$ is interpreted as the t-norm min. This entails a concrete mode of "pessimistic"computing in which, for example, a user belonging to two concepts as $\langle u: A=0.4\rangle$ and $\langle u: B=0.8\rangle$ result in $\langle u: A \sqcap B=0.4\rangle$. This behavior can be reasonable for many cases, but there exists concrete situations in which they produce inadequate results from the epistemological viewpoint. One of this cases is that of concepts or relations that are measured or estimated from others in an imprecise way. This is the case of the measure of usability of a given Web page (i.e. an hypermedia node) $\langle p:$ WebPage $=1\rangle$ that may be provided as a metadata item inside the HM. If we use a simple automated usability analysis approach [13] to obtain estimations of the usability of Web pages from quantitative indicators, a possible rule contributing to such inference may be the following:

Example Rule 3 if page is homepage and page is readable then "some conclusion"

Assuming that the estimation Readable $\sqsupset$ VeryLong $\sqcap \neg$ Dense has been defined, the antecedent of rule 3 requires the evaluation for each given page of the $\mathcal{A} \mathcal{L C}_{F H}$ concept expression as $A \equiv \neg$ VeryLong $\sqcap \neg$ Dense $\sqcap$ HomePage. Let's see a concrete example. If we have the assertions:

$\Sigma_{a}=\{\langle p:$ HomePage $=1\rangle,\langle p:$ VeryLong $=0.1\rangle,\langle p:$ Dense $=0.25\rangle\}$

following the interpretation $\mathcal{I}$ described above, the maximum degree of the antecedent $\operatorname{Maxdeg}\left(\Sigma_{a},\langle p: A\rangle\right)$ is 0.75 . The problem with this result is that it's not fully consistent with expert judgments. Expert studies point out that more complex aggregation operators are required in computing belonging to concepts that are estimated rather than defined intensionally through properties. As an illustration, the example just sketched was subject to a limited study with four 
usability experts that resulted in a set of experimental data comprising average estimations for concrete cases in the form of mappings $x_{1} \sqcap x_{2} \rightarrow y$, being $x_{1}$ and $x_{2}$ two concrete degrees $(0 \leq 0.1 \leq 0.1 \cdot 2 \leq 0.1 \cdot 3 \cdots \leq 1)$ for "not very-long" and "not dense", and $y$ the estimated appropriate resulting value. Comparing the data set with standard t-norms and t-conorms (min-max, probabilistic and Lukasiewicz), a situation like the one depicted in Figure 2 results in better adjustment properties. The rationale behind such aggregation operator design is that for lower values of both VeryLong and Dense (i.e. high values of $\neg$ VeryLong and $\neg$ Dense, delimited around the 0.7 frontier), some compensation takes place, while for higher values for both of them, the default pessimistic approach is more appropriate. The other two quadrants in Figure 2 could be represented by a non-standard function with an intermediate behavior such that $\min (x, y) \leq U(x, y) \leq \max (x, y)$.

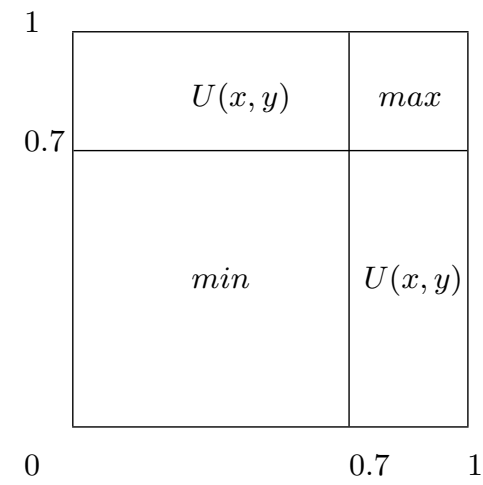

Figure 1. General scheme for $U(x, y)$

Uninorms [28] are a family of commutative, associative and monotone aggregation operators that allow such forms of compensation, having a neutral element laying anywhere in the unit interval. As such they are good candidates to generalize interpretations of conjunction and disjunction for specific cases of imperfect concept characterization. Of course, the introduction of these kinds of more flexible conjunctions as part of the DL require an analysis of its logical properties that is left to future work. It should be noted also that similar settings may appear in the case of disjunctions.

\section{Applications of Vague UI Categories to Adaptive Behaviors}

In this section some concrete applications that illustrate the concepts described in the previous section are briefly sketched, namely, preference modeling, infor- 
mation filtering problems, and imprecise adaptations on the navigational structure.

\subsection{Preference Modeling}

Preferences about UI-related descriptions can be modeled from the representations described in the previous section. These preferences may be stated in a general way as assertions in the form $\langle(u, c)$ : likesGranularity $=0.8\rangle$ where $u$ is a user of the system $\langle u: U s e r=1\rangle$, and $c$ may be a content, node (or any other form of UI element description), which can be expressed as the assertion $\langle u:$ Content $\sqcup$ Node $\sqcup$ UIElement $=1\rangle$ in a generic way. Once again, storing assertions for the whole cartesian product of users and elements may be unpractical in many situations, specially for large relations stored in databases (see [20]). We have devised an straightforward approach to granulate preferences, consisting on the representation of user preferences as related to prototypical instances representing vague categories. This way, the preference $\left\langle\left(u\right.\right.$, large $\left.-c_{i}\right)$ : likes $\left.=0.7\right\rangle$ states that the given user likes large granularity content items to the given degree.

Then rules like example rule 4 may operate on those descriptions, ultimately combining two membership mappings.

Example Rule 4 if user likes large content items and current content is $c$ then "some conclusion"

The computation will proceed by taking the degree of preference to large concepts ( 0.7 following the above example), and combining it with the degree of compatibility of the content under consideration with the vague granularity category Large (say 0.6). Then, some kind of aggregation operator (possibly flexible like the one described in the previous section) must be used to obtain the final preference. This approach may also be combined with other linguistic label aggregation operators like those described in [16], and quantified fuzzy expressions can be used also to estimate preferences as described in the same paper. For example, the rule "if most contents read by the user are short then his preference for fine granularity items is very high" can be used as an heuristic for the implicit collection of preferences.

\subsection{Information Filtering}

Imprecise preference models like those just described can be used to implement personalized filtering functions acting on UI-related criteria like those described in [16]. The abstract overall form of a filtering function is expressed in 5, where $U$ denotes the set of users, and $O$ denotes the set of the objects (in our case, information items or contents) to be filtered, resulting in a set $\mathcal{R}$ of pairs comprised by an information item and its given relevance degree. Other contextual factors (i.e. the subject of the page being read) may be added to the expression, but they're content-related, and thus out of our current scope. 


$$
\mathcal{F}: U \times O \rightarrow \mathcal{R} \text { where } \mathcal{R}=\{(o, k) \mid o \in O, k \in[0,1]\}
$$

Preferences can be used as filtering criteria, so that relevances may be obtained by computing $P_{\left(u_{i}, o_{i}\right)}=\prod_{p \in \operatorname{pref}\left(u_{i}, o_{i}\right)} p$ for a given object $o_{j}$, where pref $\left(u_{i}, o_{i}\right)$ is a set containing the values of the grades of the assertions in the form $\left\langle\left(u_{i}, o_{j}\right)\right.$ : likes $\left.X=k_{1}\right\rangle$, or $\left\langle\left(u_{i}, o-X\right)\right.$ : likes $\left.=k_{2}\right\rangle$ so that $\mu_{X}\left(o_{j}\right)>0$ (or perhaps a threshold-based version $\left.\mu_{X}\left(o_{j}\right)>w\right)$ if the more compact representation (i.e. the prototypical exemplar version) is used. Once again, the choice for the aggregator of preferences $\prod$ requires further study to be reasonable from the user viewpoint. This form of computing relevances calls for a very specific storage format for preferences, to avoid retrieving all the database of objects. One possible approach may be that of storing the likes $X$ predicates as fuzzy relations in compact $\alpha$-cut format [20] thus obtaining a $O(m+m \cdot \log m)$ complexity, being $m$ the number of relations like $X$ from $u_{i}$ having significant degrees, and provided that the relations are sorted in $m \cdot \log m$ by object to enable the computation of the final degrees in a single pass.

These UI structure-based filtering criteria can be used as a second or complementary filter, combined with content-oriented imprecise filtering expressed in fDLs, like the one sketched in [24].

\subsection{Adaptive Linking}

Hypermedia links create relationships between two or more addressable items or contents with a concrete semantic or rhetoric intention. If that intention is made explicit, it can be used by the hypermedia system to provide the link with adaptive presentation, hiding or rearranging for specific users [6]. In consequence, ontologies of link types may serve the purpose of codifying common link intentions in a formal way (e.g. as a taxonomy of link types derived from a Link concept). But a given node $n_{i}$ can be connected by link $L$ to a number of destination nodes $n_{j}$ with varying degrees of strength, as described in [21]. For example, an DetailLink may be linked with an strength of 0.8 to a full explanation of a content item, while having a link of strength 0.3 to a brief explanation. This way, the navigation structure itself becomes fuzzy, and imprecise relations can be used to implement diverse adaptations of the UI, e.g. sorting destinations by degree, or filtering only the most relevant ones. In [21], the concept of fuzzy link was proposed as a model for such interface elements, expressed in (6), where $S$ and $T$ are the sets of sources and targets (addressable entities), respectively, $U$ is the set of users, and $\Lambda$ is a set of membership functions.

$$
L=(S, T, \Lambda) \text { and } \mu_{(L, p)}:(S, T, U) \rightarrow[0,1] \text { with } \mu_{(L, p)} \in \Lambda
$$

The integration of this kind of elements in a $\mathcal{A L C}_{F H}$-like language can be carried out according to the following approach. First, links are assertions of link types subsumed by Link in $H M$. Commonly, the intention of a link is fully determined, so that assertions will be in the form $\left\langle l_{1}:\right.$ AnalogyLink $\left.=1\right\rangle$ for example. A given link will specify as sources or targets a number of nodes of 
contents also in the $H M$. These can be expressed as fuzzy or crisp assertions regarding relations, for example: $\left\langle\left(l_{1}, n_{1}\right):\right.$ source $\left.=1\right\rangle,\left\langle\left(l_{1}, n_{2}\right):\right.$ target $\left.=0.8\right\rangle$, and $\left\langle\left(l_{1}, n_{3}\right)\right.$ : target $\left.=0.3\right\rangle$. This entails a model of imprecise hypermedia structure. Personalization can be added in several ways. The simplest approach is the direct assertion of compatibility degrees of links to users, but in common cases, degrees will be computed from established mappings like (7), in which a function of the assertion denoting the (imprecise) density of the resource is combined with a function of the assertion regarding knowledge level of the user with the source $s$ to give a degree of compatibility of the link with the user (and where $s \in S, t \in T, u \in U)$.

$$
\mu_{(L, \text { detail })}(s, t, u)=f\left(\left\langle t: \text { Dense }=x_{1}\right\rangle\right) \cdot g\left(\left\langle(u, s): \text { masters }=x_{2}\right\rangle\right)
$$

Then, rules using fuzzy assertions may be used to carry out adaptations. Rule 5 describes a typical example.

Example Rule 5 if the type of the link is ArgumentLink and the link (here) is compatible with the user then font-size of the link is big and font-family of the link is Verdana

Example rule 5 provides two antecedents. On the one hand, the type of the links for which the rule is applicable is matched against assertions in the form $\langle l:$ ArgumentLink $=1\rangle$. On the other hand, the mention of the compatibility grade entails a partial match of the degree of the link. This entails a consideration of the context (i.e. sources, destinations and the current user) of the link usage, by using a predefined function definition in the form $\mu_{P}: \Sigma_{U M, D M, H M} \rightarrow[0,1]$ as the one provided in expression 7 . In addition, two kind of consequents are provided in rule 5 . The second is crisp in nature and will simply change the font type of the anchor (given that an activation threshold is reached), while the first one produce different changes depending on the activation strength. The resulting assertions make reference to personalized links (or better, link appearances), that can be denoted by $U_{i} \operatorname{Link} \doteq \operatorname{Link} \sqcap \exists$ tailored $\left\{u_{i}\right\}$ for user $i$. This way, the above rule may trigger, for example, the following two assertions: $\langle(l$, verdana $):$ fontFamily $=1\rangle$ and $\langle l:$ LargeFontSized $=0.7\rangle$ given that $l \in$ $U_{i}$ Link is the (tailored) link under consideration. The second assertion will entail a (reverse) automated assertion of the actual size in 'picas' obtained from an existing concept definition of LargeFontSized similar to those showed in Figure 1.

The just described approach can be used also to model the compatibility of users with sequences or links (paths) in the navigational structure, as a possible implementation of graph-based Web structure mining results [15].

\section{Conclusions and Future Work}

Providing handling mechanisms for vagueness in Semantic Web UI descriptions enables approximate adaptive behaviors that can't be properly addressed with 
the standard crisp semantics of DLs. This fact and the imperfection inherent to common Web user profiling techniques [23] point out the necessity of treating fuzziness as a first-class citizen in personalized Web applications. In consequence, further research should address the integration of fuzzy DLs in the common architecture of adaptive systems. The manifestations of vagueness described in this paper have been implemented in diverse scenarios, and our current work is on integrating them under a common fDL framework.

\section{References}

1. Antoniou, G.: A Nonmonotonic Rule System using Ontologies. Proceedings of the International Workshop on Rule Markup Languages for Business Rules on the Semantic Web, CEUR Workshop Proceedings, 60 (2002)

2. Baader, F., Calvanese, D., McGuinness, D., Nardi, D., Patel-Schneider, P. (eds.): The Description Logic Handbook. Theory, Implementation and Applications. Cambridge (2003)

3. Baader, F., Horrocks, I., Sattler, U.: Description Logics as Ontology Languages for the Semantic Web. In: Hutter, D, Stephan, W. (eds.): Festschrift in honor of Jörg Siekmann. Lecture Notes in Artificial Intelligence. Springer-Verlag, Berlin Heidelberg New York (2003)

4. Berners-Lee, T., Hendler, J., Lassila, O.: The Semantic Web. Scientific American, 284(5) (2001) 34-43

5. Bilgiç, T. and Türksen, T.: Measurement of Membership Functions: Theoretical and Empirical Work. In: D. Dubois and H. Prade (eds.) Handbook of Fuzzy Sets and Systems Vol. 1, Chapter 3, Fundamentals of Fuzzy Sets, Kluwer (1999):195-232

6. Brusilovsky, P. (2001) Adaptive hypermedia. User Modeling and User Adapted Interaction, Ten Year Anniversary Issue (Alfred Kobsa, ed.) 11(1/2), 87-110

7. Brusilovsky, P. and Maybury, M. T.: From adaptive hypermedia to adaptive Web. Communications of the ACM 45 (5), (2002) 31-33

8. Dodero, J.M., Sicilia, M.A., García, E.: A Fuzzy Aggregation-Based Reputation Model for e-Learning Exploitation of Public Domain Resources. In: Proceedings of the Fourth International ICSC Symposia on Soft-Computing And Intelligent Systems For Industry ICSC Naiso Academia Press, Paisley, Scotland (2001)

9. García, E., Sicilia, M.A., Gutiérrez, J.A.: On the Vague Modelling of Web Page Characteristics Regarding Usability. In: Menasalvas, E., Segovia, J., Szczepaniak, P. (eds.): First International Atlantic Web Intelligence Conference. Lecture Notes in Computer Science, Vol. 2663. Springer-Verlag, Berlin Heidelberg New York (2003) 199-207

10. Glöckner, I. and Knoll, A.: A Framework for Evaluating Fusion Operators Based on the Theory of Generalized Quantifiers. In: Proceedings of the 1999 IEEE International Conference on Multisensor Fusion and Integration for Intelligent Systems (MFI '99), Taipei, Taiwan (1999)

11. Guarino, N.: Formal ontology, conceptual analysis and knowledge representation. Int. Journal of Human-Computer Studies, 43(5/6) (1995) 625-640

12. Hölldobler, S., Dinh Khang, T., Störr, H.P.: A fuzzy description logic with hedges as concept modifiers. In: Hoang Phuong, N., Nguyen, H.T., Cat Ho, N., Santiprabhob, P. (eds.): Proceedings of InTech/VJFuzzy'2002, Hanoi, Vietnam, (2002) 25-34

13. Ivory, M.Y. and Hearst, M.A.: The State of the Art in Automated Usability Evaluation of User Interfaces. ACM Computing Surveys, 33(4) (2001):147 
14. Ivory, M.Y. and Hearst, M.A.: Improving Web Site Design. IEEE Internet Computing, Special Issue on Usability and the World Wide Web 6(2), March/April (2002): $56-63$

15. Kosala, R., Blockeel, H.: Web mining research : A survey. In: SIGKDD Explorations - Newsletter of the ACM Special Interest Group on Knowledge Discovery and Data Mining 2(1) (2000) 1-15

16. López, L., Sicilia, M.A., García, E.: Personalization of Web Interface Structural Elements: A Learning-Scenario Case Study. In: International Symposia of Computer Science. Aguascalientes, Mexico (2001) 579-588

17. McCarthy, J.: Epistemological problems of artificial intelligence. In: Proceedings Int. Joint Conference on Artificial Intelligence (1997) 1038-1044

18. Rosch, E. Principles of Categorization. In: Rosch, E., Lloyd, B. (eds.), Cognition and Categorization, Lawrence Erlbaum, Hillsdale, NJ (1978) 27-48

19. Sicilia, M. A., Díaz, P., Aedo, I., García, E.: Fuzzy Linguistic Summaries in Adaptive Hipermedia Systems. In: Adaptive Hipermedia and Adaptive Web Systems. Lecture Notes in Computer Science, Vol. 2347, Springer-Verlag, Berlin Heidelberg New York (2002) 317-327

20. Sicilia, M.A., Gutiérrez, J.A., García, E.: Designing Fuzzy Relations in Orthogonal Persistence Object-Oriented Database Engines. Advances in Artificial Intelligence - IBERAMIA 2002, Lecture Notes in Computer Science 2527 Springer (2002) 243253

21. Sicilia, M. A., García, E., Díaz, P. Aedo, I.: Learning Links: Reusable Assets with Support for Vagueness and Ontology-based Typing. In: International Workshop on Concepts and Ontologies in Web-based Educational Systems. Aroyo, L., Dicheva, D. (eds.):Technical Report 02-15, Technical University of Eindhoven (2002) 37-42

22. Sicilia, M.A., García, E., Díaz, P., Aedo, I.: Fuzziness in adaptive hypermedia models. In: Proceedings of the North American Fuzzy Information Processing Society Conference (2002) 268-273

23. Sicilia, M.A.: ObservingWeb Users: Conjecturing and Refutation on Partial Evidence In: Proceedings of the North American Fuzzy Information Processing Society Conference (2003)

24. Straccia, U.: A Framework for the Retrieval of Multimedia Objects Based on FourValued Fuzzy Description Logics. In: Crestani, F., Pasi, G. (eds.): Soft Computing in Information Retrieval: Techniques and Applications. Physica Verlag (Springer Verlag), Vol. 50. Heidelberg, Germany, (2000) 332-357

25. Straccia, U.: Reasoning within fuzzy description logics. J. Artificial Intelligence Research, 14 (2001) 137-166.

26. Tresp, C.B., Molitor, R.: A Description Logic for Vague Knowledge. In: Proceedings of the 13th biennial European Conference on Artificial Intelligence (ECAI'98), J. Wiley and Sons, Brighton, UK (1998) 361-365

27. Wu, H., De Kort, E., De Bra, P.: Design Issues for General-Purpose Adaptive Hypermedia Systems. In: Proceedings of the ACM Conference on Hypertext and Hypermedia (2001) 141-150

28. Yager, R. R., and Rybalov, A.: Uninorm Aggregation Operators. Fuzzy Sets and Systems 80 (1996) 111-120

29. Zadeh, L.A.: A Computational Approach to Fuzzy Quantifiers in Natural Language. Computing and Mathematics with Applications, 9(1) (1983) 149-184 